\title{
Ultrasound-assisted transthoracic biopsy: fine-needle aspiration or cutting-needle
} biopsy?

\author{
A.H. Diacon*, J. Theron*, P. Schubert" , K. Brundyn\#, M. Louw”, \\ C.A. Wright ${ }^{\#}$ and C.T. Bolliger*
}

ABSTRACT: The present study compared the diagnostic yield of ultrasound-assisted cuttingneedle biopsy (CNB) and fine-needle aspiration biopsy (FNAB) in chest lesions.

A physician performed ultrasound and FNAB with a 22-G spinal needle in all patients, directly followed by a 14-G CNB in patients without contraindication.

A total of 155 consecutive lesions arising from the lung (74\%), pleura (12\%), mediastinum (11\%) or chest wall $(3 \%)$ in patients with a final diagnosis of lung carcinoma $(74 \%)$, other malignant tumours $(12 \%)$, non-neoplastic disease $(9 \%)$ or unknown $(5 \%)$ were prospectively included. The overall diagnostic yield was $87 \%$. Combined specimens were obtained in 123 lesions (79\%). In these, yields of FNAB, CNB and both methods combined were 82, 76 and $89 \%$, respectively. FNAB was significantly better than CNB in lung carcinoma (95 versus $81 \%$ ) but CNB was superior in noncarcinomatous tumours and in benign lesions. On-site cytology was $\mathbf{9 0 \%}$ sensitive and $\mathbf{1 0 0 \%}$ specific for predicting a positive FNAB. One patient required drainage for pneumothorax (0.6\%).

Ultrasound-assisted fine-needle aspiration biopsy performed by chest physicians is an accurate and safe initial diagnostic procedure in patients with a high clinical probability of lung carcinoma. All other patients should undergo concurrent fine-needle aspiration biopsy and cutting-needle biopsy.

KEYWORDS: Cutting-needle biopsy, fine-needle aspiration, lung biopsy, lung carcinoma, pleural biopsy, ultrasound

$\mathbf{U}$ ltrasound (US) has found a firm place in chest medicine as an aid for assessing pleural effusions at the bedside [1]. This development was facilitated by the advent of affordable, lightweight and mobile US units. Although less practised by physicians, US can also visualise solid lesions arising from the pleura, chest wall and anterior mediastinum, and even lung tumours and consolidations are detected without difficulty provided they extend to the parietal pleura. US is an ideal tool to assist with biopsy procedures. It can frequently replace computed tomographic (CT) guidance at much lower cost [2].

US-assisted cutting-needle biopsy (CNB) performed by chest physicians was $100 \%$ sensitive for mesothelioma in a recent prospective study carried out at the present authors' institution [3]. In that study, the majority of candidates for USassisted biopsy did not suffer from pleural disease but presented with peripheral lung carcinoma, for which fine-needle aspiration biopsy (FNAB) might be a safer and technically easier alternative to CNB. US-assisted FNAB integrates easily with routine practice, as it only requires basic ultrasound equipment and consumables commonly used by chest physicians for diagnostic thoracentesis or lymph node aspiration. FNAB and CNB both have a high diagnostic yield for lung carcinoma under CT guidance [4, 5], which is essential for lung lesions located within the lung parenchyma. In contrast, US can only be used on lesions with pleural contact, which frequently represent rather large and partly necrotic lung tumours, inflammatory processes or consolidations due to central bronchial obstruction. It is unknown which biopsy device performs better in chest lesions accessible to US. The present study directly compared the diagnostic yield of US-assisted transthoracic FNAB and CNB.

\section{PATIENTS AND METHODS}

All patients referred to the Lung Unit of Tygerberg Hospital (Cape Town, South Africa;

\section{AFFILIATIONS}

${ }^{*}$ Depts of Internal Medicine and

\#Anatomical Pathology, Tygerberg Academic Hospital and National Health Laboratory Service, University of Stellenbosch, Cape Town, South Africa.

\section{CORRESPONDENCE}

A.H. Diacon

Dept of Internal Medicine PO Box 19063

7505 Tygerberg

South Africa

Fax: 27219317442

E-mail: ahd@sun.ac.za

Received:

June 142006

Accepted after revision:

0ctober 222006

SUPPORT STATEMENT

A.H. Diacon was supported by a grant of the University of Stellenbosch. The authors thank the Holland Stellenbosch Medical Foundation, Veldhoven, The Netherlands, for their continued support.

STATEMENT OF INTEREST None declared. 
tertiary academic centre, catchment area 1.5 million) with lesions on chest radiography or $\mathrm{CT}$ suspected to involve the chest wall or the pleura were candidates for the present study. Patients with pleural effusions were not enrolled but were further evaluated with pleural fluid cytology or thoracoscopy. A consultant respiratory physician or a registrar under supervision performed sonography (Toshiba Sonolayer L SAL 77A, 3.75-MHz sector probe; Toshiba, Johannesburg, South Africa) and biopsy in free-hand technique (no dedicated biopsy sonar probe) in a bronchoscopy suite. All patients gave signed informed consent. Ethical approval from the institutional review board was obtained.

\section{Biopsy procedure}

After consulting the radiological evidence the target lesion was identified with US and the most suitable patient position, as well as entry site, direction and depth for the biopsy, were determined. Under local anaesthesia with lidocaine $1 \%$, the lesion was first aspirated with a 22-G spinal needle (38 or $90 \mathrm{~mm}$; Becton Dickinson, Madrid, Spain) connected to a 10-mL syringe. Three passes from slightly different areas of the target were directly expressed onto slides and smeared. The slides were either air dried or submitted for rapid on-site analysis (ROSE) using Diff-Quik (Rapidiff; Clinical Sciences Diagnostics, Southdale, South Africa) or rapid Papanicolaou staining methods [6]. Immediately thereafter, two or more CNB passes were performed at the same location until macroscopically satisfactory material was harvested (14-G cutting needle, manually driven, specimen notch $20 \mathrm{~mm}$; Allegiance, Chateaubriand, France). To ensure a consistent effort with the $\mathrm{CNB}$ procedure, the operator was only informed about the ROSE result upon completion of the CNB. When the lesions were small ( $<3 \mathrm{~cm}$ in diameter), mobile, in close vicinity to vital structures, or when a patient was in poor medical condition, it was left to the physician to decide whether or not to perform a $\mathrm{CNB}$. The reason for not performing CNB was documented by the physician. Directly after the procedure, the site was reexamined with US. A follow-up chest radiograph was obtained if the pre- and post-biopsy US findings differed and at the discretion of the physician.

\section{Assessments and measurements}

Lesions were classified as follows on radiological appearance as to the most likely origin: "pleural" (pleural based, blunt angle to the lung), "pulmonary" (lesion with centre in the lung, acute angle to the pleura), "chest wall" (lesions centred in the chest wall with pleural involvement) or "mediastinal" (lesions predominantly located in the anterior mediastinum with extension to the pleura). The maximum depth of the target lesion was measured sonographically in the direction of the planned puncture. If the target extended deeper than $100 \mathrm{~mm}$ from the skin, $100 \mathrm{~mm}$ was noted. As an indication for locally advanced disease, the presence of superior vena cava syndrome or signs of direct spread into nerval tissue (nerve roots, spinal cord, brachial plexus) was recorded. This would indicate surgical nonresectability in the setting of nonsmall cell lung carcinoma.

\section{Scoring and statistical analysis}

Specimens with an unequivocal diagnosis of neoplastic disease or a positive stain or culture for mycobacteria were accepted as diagnostic. Nondiagnostic specimens contained normal cells or tissue, nonrepresentative material, or necrotic cells or tissue. Typing discrepancies between nonsmall cell lung cancer (NSCLC) subgroups were not considered typing errors because dual differentiation is common and it is not of therapeutic relevance. The accuracy of ROSE for predicting diagnostic material on-site was established by comparison with the final FNAB result. Patients with nondiagnostic FNAB and CNB were either clinically observed or further investigated by the appropriate means. Two different pathologists independently reviewed FNAB and CNB specimens. Descriptive statistics and Chi-squared comparisons of proportional data were performed. A p-value $<0.05$ in a two-tailed test of proportions (Chi-squared) was considered significant.

\section{RESULTS}

\section{Patients and lesions}

A total of 155 patients were included over a 23-month period. Median age was 56 yrs (range 27-90 yrs), 68\% were male and $50 \%$ were outpatients. The majority of cases were lung lesions extending to the pleura $(n=115,74 \%)$, followed by pleuralbased lesions $(n=18,12 \%)$, mediastinal lesions $(n=17,11 \%)$ and lesions situated in the chest wall $(n=5,3 \%)$. At the time of biopsy, a chest radiograph was available in all patients and a chest CT scan in 100 patients (64\%). Locally advanced disease with clinical signs of superior vena cava compression was noted in 20 patients $(13 \%)$ and invasion of spinal or plexus nerval tissue in $12(8 \%)$. Mean maximum lesion depth from the skin was $73 \mathrm{~mm}$ (range $20-100 \mathrm{~mm}$ ).

\begin{tabular}{|c|c|c|}
\hline \multirow[t]{2}{*}{ TABLE 1} & \multicolumn{2}{|c|}{$\begin{array}{l}\text { yield of fine-needle aspiration } \\
\text { and cutting-needle biopsy (CNB) }\end{array}$} \\
\hline & $\begin{array}{c}\text { Final } \\
\text { diagnosis }\end{array}$ & $\begin{array}{l}\text { Diagnostic FNAB } \\
\text { and/or CNB }\end{array}$ \\
\hline All & $155(100)$ & $135(87)$ \\
\hline Malignant lesions & $133(86)$ & $126(95)$ \\
\hline Lung carcinoma & $114(74)$ & $110(96)$ \\
\hline Small cell carcinoma & $11(7)$ & $11(100)$ \\
\hline Adenocarcinoma & $37(24)$ & $37(100)$ \\
\hline Squamous cell carcinoma & $16(10)$ & $16(100)$ \\
\hline Undifferentiated carcinoma & $50(32)$ & $46(92)$ \\
\hline Sarcoma & $8(5)$ & $8(100)$ \\
\hline Lymphoma & $5(3)$ & $2(40)$ \\
\hline Mesothelioma & $2(1)$ & $2(100)$ \\
\hline Other malignant ${ }^{\#}$ & $4(3)$ & $4(100)$ \\
\hline Benign lesions & $14(9)$ & $9(64)$ \\
\hline Fibrosis & $6(4)$ & $2(33)$ \\
\hline Tuberculosis & $6(4)$ & $5(83)$ \\
\hline Lipoma & $1(1)$ & $1(100)$ \\
\hline Wegener's granulomatosis & $1(1)$ & $1(100)$ \\
\hline Undiagnosed & $8(5)$ & \\
\hline
\end{tabular}




\section{TABLE 2 Cases with negative biopsy procedures}

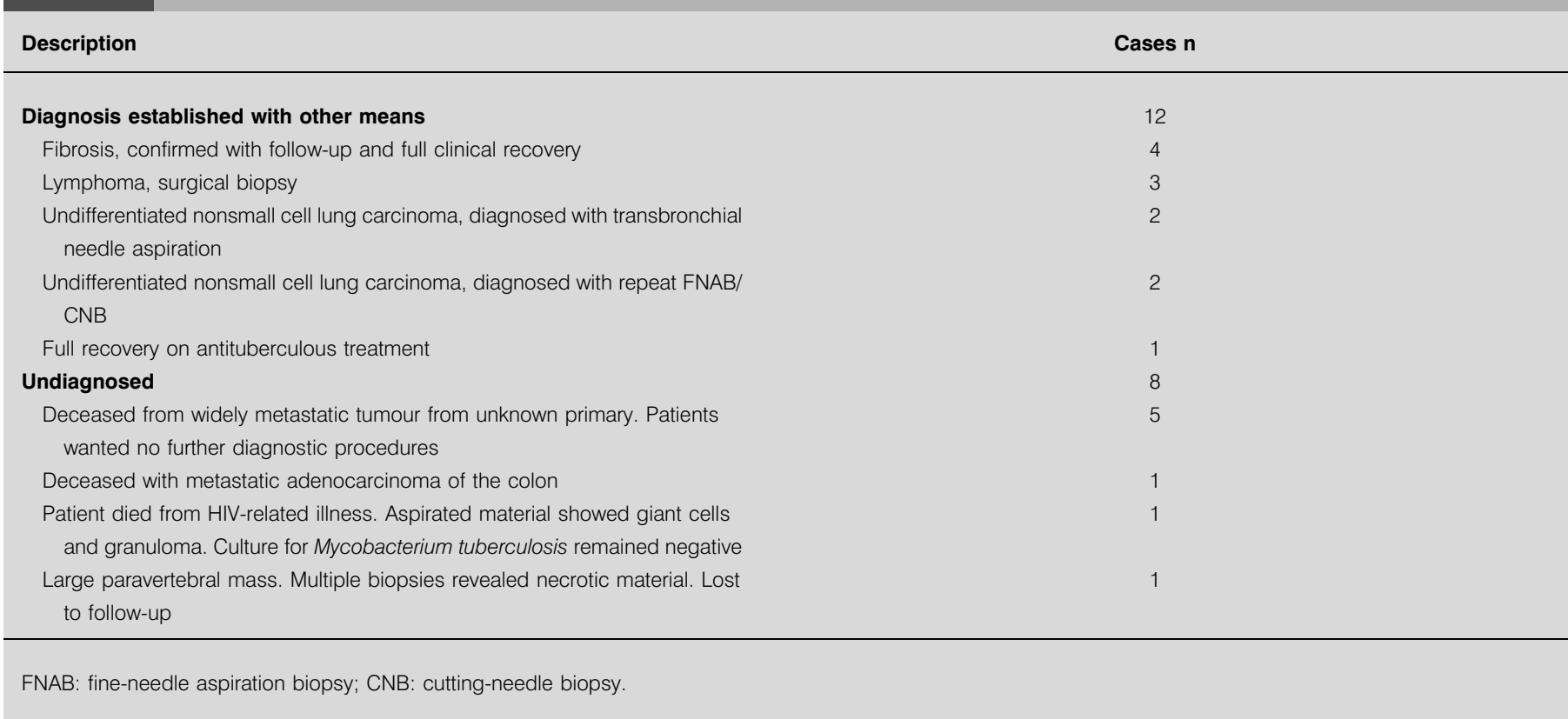

\section{Diagnosis, procedures and safety}

A final diagnosis was established in 147 patients (95\%). Most diagnoses were established with US-assisted CNB and/or FNAB (135 patients, $87 \%$; table 1$)$. Twelve patients (8\%) were diagnosed by other means and eight patients $(5 \%)$ remained undiagnosed (table 2). The physician decided not to perform CNB in 32 patients $(21 \%)$ because of technical or patientrelated reasons (table 3 ). The diagnostic yield in these patients was $81 \%$ (malignant lesions $92 \%$; benign lesions 57\%). Adverse events did not occur in patients undergoing FNAB only, and were infrequent and mostly minor in those with combined procedures. Pneumothorax was observed in two patients $(1.3 \%)$, and in one of these, a chest drain was required $(0.6 \%)$. Mild puncture-site bleeding $(n=4,2.6 \%)$, post-procedural pain requiring medication $(n=2,1.3 \%)$, vagovasal reaction $(n=2,1.3 \%)$ and transient mild haemoptysis $(n=1$, $0.6 \%$ ) were minor events.

\section{Histological diagnosis and correlation in paired samples}

Paired diagnostic specimens (FNAB and CNB) were obtained in 123 patients $(79 \%)$ with a combined diagnostic yield of
$89 \%$ (table 4 ). The yield of FNAB and CNB alone was 82 and $76 \%$, respectively (difference not significant). FNAB had a significantly higher yield than CNB in neoplastic disease (91 versus $82 \%, \mathrm{p}=0.05)$, mainly due to a higher yield in lung carcinoma (95 versus $81 \%, \mathrm{p}=0.006$ ). Only one case of lung carcinoma (1\%) was identified exclusively by CNB, whereas 14 cases $(15 \%)$ were diagnosed with FNAB but not with CNB. Most of these tumours were of larger size than average and all showed necrosis on histology and/or necrotic areas on CT. Cytology and histology were concordant for SCLC and NSCLC in 74 out of 75 cases (99\%). One case diagnosed as adenocarcinoma on cytology was classified as an atypical carcinoid on histology with immunohistochemistry, and this was later confirmed on resection of the tumour. This change in final diagnosis did not affect the management in that particular patient as surgical resection was the therapy of choice for both tumour types. When a diagnosis other than lung carcinoma was established $(n=22)$, CNB was superior to FNAB with 18 versus 12 positive cases (82 versus 55\%, $\mathrm{p}=0.056$ ). However, in eight out of 12 cases with positive FNAB the definite classification was only possible with the additional information

TABLE 3 Reasons for not performing cutting needle biopsy in 32 patients

Patients $\mathbf{n}$

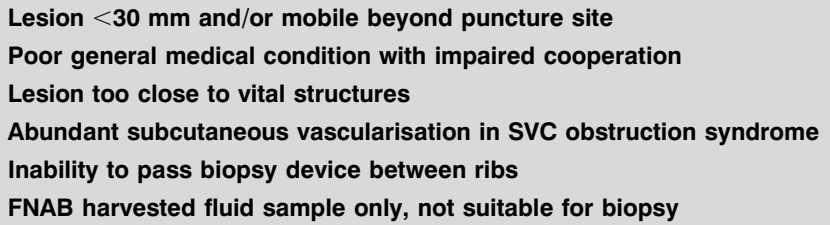




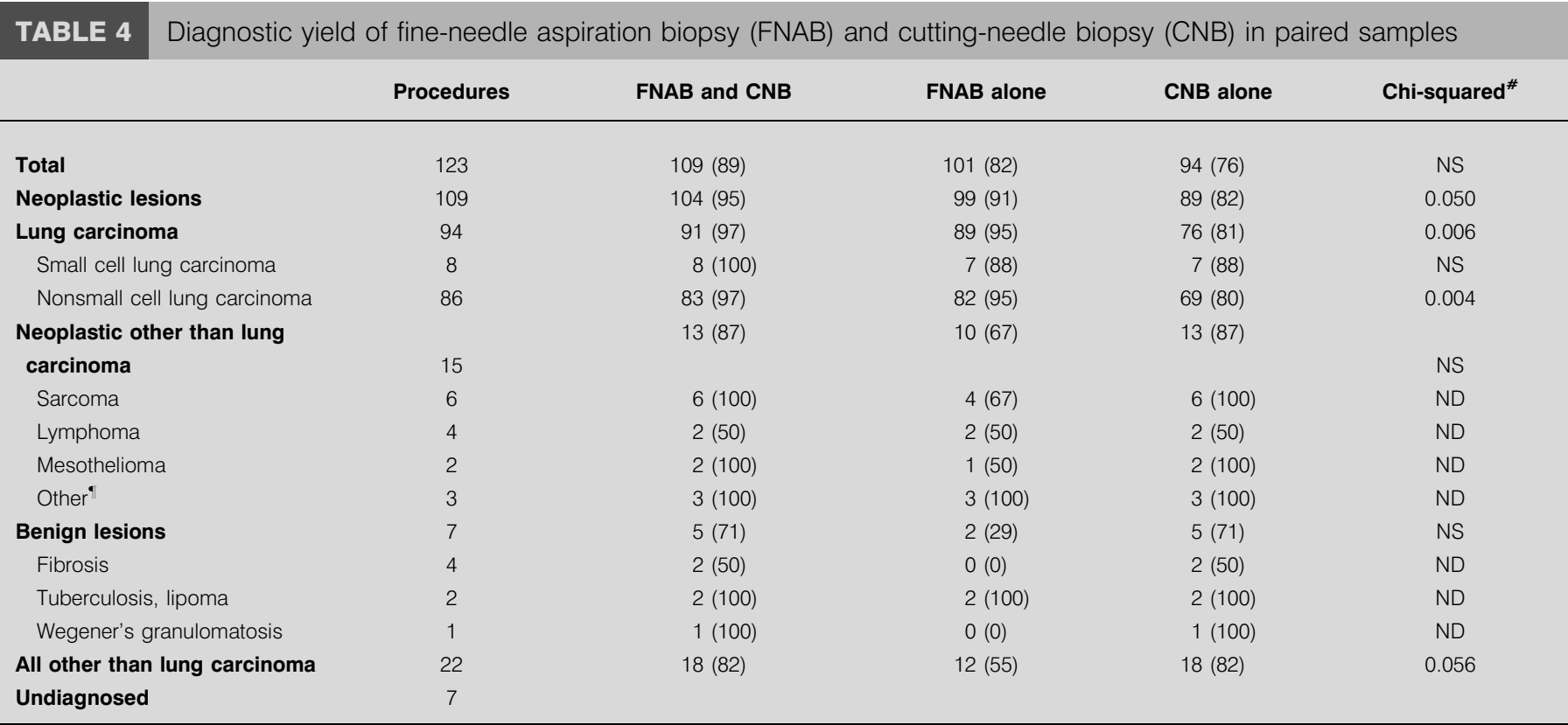

Data are present as $n$ and $n(\%)$, unless otherwise stated. ND: not done. NS: nonsignificant. ${ }^{\#}$ : Chi-squared, FNAB versus CNB; $p$-values $\leqslant 0.1$ are displayed, others are NS; ${ }^{\prime}$ : Thymoma, carcinoma with unknown primary, carcinoid tumour (diagnosed as adenocarcinoma on FNAB)

from the CNB specimen (sarcoma $n=4$; lymphoma $n=2$; mesothelioma $n=1$; thymoma $n=1$ ). ROSE was $90 \%$ sensitive and $100 \%$ specific for predicting the presence of diagnostic material in positive FNAB.

\section{DISCUSSION}

The main finding of this prospective comparison of USassisted FNAB and CNB in 155 chest lesions of mixed origin is the high combined diagnostic yield (87\%), with a low rate of complications. FNAB and CNB are complementary techniques. Out of all the malignant tumours, $18 \%$ were diagnosed with only one modality. The yield of FNAB alone was significantly higher in lung carcinoma, while CNB was superior in all other malignant tumours or in benign diseases. The high yield (95\%) of US-assisted FNAB in 89 cases of lung carcinoma is clinically relevant. Chest pain indicating pleural involvement is a common presenting symptom of lung caranoma, and a considerable proportion of these patients might qualify for US-assisted FNAB. This procedure is safe, can be performed at the bedside by a chest physician using the same tools as for a pleural tap or a diagnostic lymph node aspiration, and might be preferable to the more expensive and less convenient CTguided approach in many cases.

Compared with the current authors' previous series of 91 USassisted CNB [3], the high diagnostic yield has been maintained with a reduced pneumothorax rate. This is most probably due to the fact that FNAB replaced CNB in situations deemed by the investigator to carry an elevated risk for pneumothorax. It is reasonable to assume that FNAB causes less tissue damage than CNB and is therefore an inherently safer procedure. Small lesions, particularly when mobile on respiration (fig. 1), are at risk of being punctured slightly off centre. The chance of causing pneumothorax by accidental puncture of aerated tissue increases with the size of the biopsy device. Its safety, combined with its superior yield in lung carcinoma, makes US-assisted FNAB the first choice for biopsy of lesions originating from the lung. In contrast, lesions arising from the chest wall, pleura or mediastinum are, by their nature, safe targets for CNB because they are not mobile on respiration and are not surrounded by lung tissue. Such lesions are also less likely to represent carcinomatous tumours, which favour CNB due to the better yield in comparison to FNAB. As an added advantage, FNAB specimens are suited for ROSE, which is of proven value in transbronchial needle aspiration [7, 8], as well as in CT-guided aspiration of chest lesions [9, 10]. The sensitivity of ROSE for the presence of diagnostic material was $90 \%$ in the present study.

The superior yield of FNAB in lung carcinoma in the present study is intriguing. FNAB did not have a better yield than $\mathrm{CNB}$ in lung carcinomas in the large series by GONG et al. [4] reporting on CT-guided biopsies. This may partly be due to selection bias, because GoNG et al. [4] only performed CNB when FNAB smears were considered suboptimal on immediate on-site assessment. Another explanation for the high rate of false-negative CNB could lie in the nature of neoplastic lung lesions detectable on US. Such lesions often show a nonhomogenous picture with a mixture of necrotic areas, vital tumour and atelectatic lung tissue. A CNB pass can harvest a sizeable specimen from one area of the lesion, but this might only contain nonrepresentative material [5]. Moreover, scarce vital tumour tissue might easily be missed because only a small portion of a histological sample can routinely be cut and analysed under the microscope. In contrast, a fine needle passed through a lesion repeatedly can sample a 

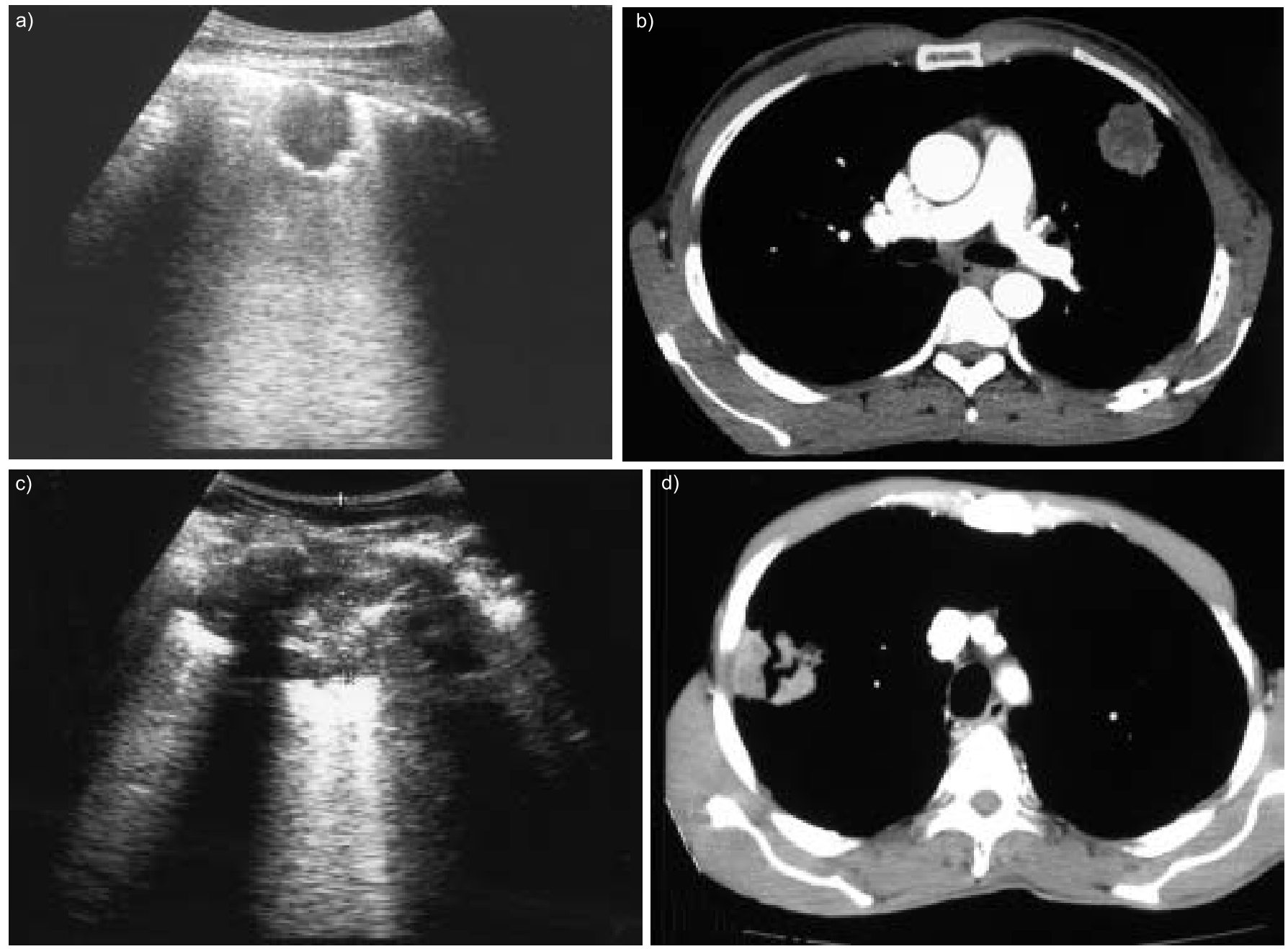

FIGURE 1. a) Sonographic image and b) computed tomography (CT) of a subpleural pulmonary tumour ( $40 \times 30 \mathrm{~mm}$ on CT) that showed marked movement on respiration. The small area of contact with the pleura made its location with ultrasound (US) difficult. This patient only underwent fine-needle aspiration biopsy (FNAB). The diagnosis was adenocarcinoma. c) Sonographic image and d) CT of a lesion of similar size (40 $\times 30 \mathrm{~mm}$ on CT), arising from the lung and adherent to the pleura. The lesion showed no movement on respiration and the area of contact with the pleura was comparatively large. This lesion was easy to locate with US. This patient underwent FNAB and cutting biopsy without complications. The diagnosis was large cell lung carcinoma on both specimens.

comparatively large area. The specimen is less voluminous and can be completely scrutinised after direct transfer onto slides. This makes a positive result possible even when few vital cells have been collected. Tissue quality could also explain why CNB has a higher yield in nonepithelial malignant neoplasms. These tend to be homogenous and their high intercellular adherence makes aspiration difficult [4]. CNB specimens also facilitate the assessment of histological architecture and the performance of ancillary studies, which is often essential for classifying such tumours [4].

Despite similarly high yields, ultrasound-assisted fine-needle aspiration biopsy and cutting-needle biopsy are complementary methods for establishing a tissue diagnosis in patients with chest tumours involving the pleura. Considering its low degree of invasiveness, its simplicity, safety and low cost, ultrasound-assisted fine-needle aspiration biopsy is the method of choice in all patients with a high clinical probability of lung carcinoma. All other patients should undergo concurrent ultrasound-assisted fine-needle aspiration biopsy and cutting-needle biopsy.

\section{REFERENCES}

1 Beckh S, Bolcskei PL, Lessnau KD. Real-time chest ultrasonography: a comprehensive review for the pulmonologist. Chest 2002; 122: 1759-1773.

2 Diacon AH, Theron J, Bolliger CT. Transthoracic ultrasound for the pulmonologist. Curr Opin Pulm Med 2005; 11 : 307-312.

3 Diacon AH, Schuurmans MM, Theron J, Schubert PT, Wright CA, Bolliger CT. Safety and yield of ultrasoundassisted transthoracic biopsy performed by pulmonologists. Respiration 2004; 71: 519-522.

4 Gong Y, Sneige N, Guo M, Hicks ME, Moran CA. Transthoracic fine-needle aspiration ws. concurrent core needle biopsy in diagnosis of intrathoracic lesions: a retrospective comparison of diagnostic accuracy. Am J Clin Pathol 2006; 125: 438-444. 
5 Greif J, Marmur S, Schwarz Y, Man A, Staroselsky AN. Percutaneous core cutting needle biopsy compared with fineneedle aspiration in the diagnosis of peripheral lung malignant lesions: results in 156 patients. Cancer 1998; 84: 144-147.

6 Keebler KM. Cytopathology techniques. In: Bibbo M, ed. Comprehensive Cytopathology. 2nd Edn. Philadelphia, WB Saunders, 1997; pp. 881-906.

7 Davenport RD. Rapid on-site evaluation of transbronchial aspirates. Chest 1990; 98: 59-61.
8 Diacon AH, Schuurmans MM, Theron J, et al. Utility of rapid on-site evaluation of transbronchial needle aspirates. Respiration 2005; 72: 182-188.

9 Conces DJ Jr, Schwenk GR Jr, Doering PR, Glant MD. Thoracic needle biopsy. Improved results utilizing a team approach. Chest 1987; 91: 813-816.

10 Austin JH, Cohen MB. Value of having a cytopathologist present during percutaneous fine-needle aspiration biopsy of lung: report of 55 cancer patients and metaanalysis of the literature. AJR Am J Roentgenol 1993; 160: 175-177. 